\title{
Higgs Scalar-Tensor Theory for Gravity and the Flat Rotation Curves of Spiral Galaxies
}

\author{
Nils M. BEZARES-Roder ${ }^{1}$, HeINZ DEHNEN ${ }^{2}$ \\ 1. Institut für Theoretische Physik, Universität Ulm 1 \\ 2. Fachbereich Physik, Universität Konstan 2 \\ DOI $10.1007 / \mathrm{s} 10714-007-0449-8$ \\ General Relativity and Gravitation 39(8), 1259-1277 (2007)
}

\begin{abstract}
The scalar-tensor theory of gravity with the Higgs field as scalar field is presented. For central symmetry it reproduces the empirically measured flat rotation curves of galaxies. We approximate the galaxy by a polytropic gas sphere with the polytropic index $\gamma=2$ and a massive core.
\end{abstract}

\section{Introduction}

Usually, flat rotation curves of spiral galaxies are interpreted in such a way that the galaxies contain a significant quantity of non-luminous (dark) matter (DM), which leads to a great mass discrepancy $D=\frac{M_{d y n}}{M_{o b s}}-1$ between the luminous -observed- and dynamical mass of a galaxy. Another interpretation is that the usual Newtonian inverse square law of gravity has to be modified on large scales.

Currently, the nature and the distribution of dark matter is unknown. It is suggested that a spiral galaxy is surrounded by a spherical halo of cold dark matter (CDM), the extension of which is much greater than the visible disk [22]. This idea is determined from measurements of the $21-\mathrm{cm}$ line of neutral hydrogen outside the region of the optical radius. Additionally, these measurements point out that there 'exists a sensitive coupling between visible and dark matter [1] (disc-halo conspiracy). Today, the dark matter hypothesis is generally accepted, mainly due to the fact that changing established physical laws is usually avoided.

Nevertheless, the Newtonian $r^{-2}$-law is only empirically tested in the satellite region and the solar system, so that flat rotation curves might be a first hint for its failing on large scales $(\geq 20 \mathrm{kpc})$. In this context, several modifications of the Newtonian law have been proposed, but nearly all of them are constructed ad hoc.

One important attempt of modification is the FLAG (finite length-scale anti-gravity)-theory of Sanders [24], who added a Yukawa potential with finite length scale $r_{0}$ and a negative coupling constant $\hat{\alpha}$ to the usual gravitational potential

$$
\Phi=-\frac{G_{\infty} M}{r}\left(1+\hat{\alpha} e^{-r / r_{0}}\right),
$$

where $G_{\infty}$ is the value of the gravitational constant at infinity, whereas the local value is variable. For $\hat{\alpha} \approx-0,92$ and $20 \mathrm{kpc}<r_{0}<40 \mathrm{kpc}$, the model reproduces the rotation curves of galaxies ranging in size from 5 to $40 \mathrm{kpc}$.

Milgrom [21] found that the deviation from the Newtonian law in large astronomical systems apparently

\footnotetext{
${ }^{1}$ Albert-Einstein-Allee 11, D-89069 Ulm. E-mail: Nils.Bezares@uni-ulm.de

${ }^{2}$ Fach M677 Fachbereich Physik, D-78457 Konstanz. E-mail: Heinz.Dehnen@uni-konstanz.de

${ }^{3}$ The original publication is available at www.springer.com.
} 
appears below a critical acceleration. In his modified Newton dynamics (MOND) the equation of motion becomes

$$
m \mu\left(a / a_{0}\right) \vec{a}=\vec{F}
$$

where $a_{0}$ is a critical acceleration and $\mu\left(a / a_{0}\right)$ is a function determined from observations and having the asymptotical behaviour $\mu\left(a / a_{0}\right)=a / a_{0}$ for $a \leq a_{0}$ and $\mu\left(a / a_{0}\right)=1$ for $a \gtrsim a_{0}$. Equation (2) leads to acceptable results but does not have a deeper theoretical foundation. Therefore, Beckenstein and Milgrom [2] tried to formulate the MOND within a field theory. They started from a non-relativistic Lagrangian, which leads to a modified Poisson equation. In this way they can describe mass discrepancies in low accelerated systems.

Additionally, there exist other field theories of gravity that modify even the classical General Relativity by the addition of one or more scalar fields to the tensor field of General Relativity. This kind of general relativistic models with a scalar field are conform equivalent with more-dimensional general relativistic models [7]. The first such attempt was started by Jordan [18]. He noticed through his isomorphy theorem that projective spaces as Kaluza-Klein's can be reduced to usual Riemannian 4-dim spaces and that a scalar field as fifth component of such a projective metric can play the role of a variable gravitational "constant", by which it is possible to vary the strength of gravitation [15].

In his theory, Jordan introduced two coupling parameters of the scalar field, one producing a variation of the gravitational constant and another one that would break the energy conservation through a nonvanishing divergence of the energy-stress tensor to increase the mass in time, in accordance with the ideas of Dirac [19]. However, the microwave background radiation as a real black-body radiation discovered in 1965 forces us to accept general energy conservation as given [17]. Jordan's theory was worked out independently by Brans and Dicke [4] without changing energy conservation, but again introducing a scalar field with an infinite length scale and playing the role of a variable gravitational coupling. This time it followed Mach's principle of the relativity of inertia, that with respect to the equivalence principle, the inertial, as well as passive and active gravitational mass should be induced by the interaction with the gravitational field [13]. O. Obregón investigated in that subject beginning in the 1970's. The only coupling parameter $\omega$ of this scalar-tensor theory is a measure of the strength of the scalar field coupling to matter. For a sensitive theory, it should be of the order of unity [4]. For $\omega \rightarrow \infty$, General Relativity is obtained, and, in fact, solar system experiments restrict $\omega$ to be greater than about 500 [23], which entails that the Brans-Dicke theory leads nearly to the same results as General Relativity.

On the other hand, in the elementary particle physics the inertial as well as passive gravitational masses are generated simultaneously with respect to gauge invariance by the interaction with a scalar Higgs field through the so-called Spontaneous Symmetry Breakdown. Because of the identity of passive and active gravitational mass also the latter, i.e. the gravitational constant should be produced by the same symmetry breaking. Additionally, Dehnen et al. [8, 9] showed that the interaction of the Higgs field with the particles which gain mass through it is of a gravitational and Yukawa nature, so that they finally identified the Higgs field with that of the scalar-tensor theory of gravity possessing a variable gravitational "constant" [10]. In such a theory, the Newtonian gravitational constant as well as the elementary particle masses can be generated simultaneously by breaking symmetry. Here, the scalar field has a finite length scale, which causes a Yukawa-like potential similar to the one in Eq.(1), and following the ideas of Zee [26], as a broken-symmetric theory of gravity.

Gessner showed [16] that a negative cosmological constant $\Lambda$ gives rise to flat rotation curves that would, however, reduce the age for the universe too much. Nevertheless, the scalar-tensor theory of Dehnen et al. contains a cosmological function $\Lambda(\varphi)$ (instead of a constant), proportional to the Higgs potential $V(\varphi)$, and therefore, the above-mentioned problem may not appear using this theory. Also the nature and value of the cosmological "constant" may be understandable through it. The first such attempt within the HSTT was made by Cervantes-Cota and Dehnen [5, 6] for an explanation of inflation. 


\section{Higgs scalar-tensor theory of gravity}

The model starts with a Lagrange density in unique form $(\hbar=1, c=1)^{4}$ :

$$
\mathcal{L}=\left[\frac{1}{16 \pi} \breve{\alpha} \phi^{\dagger} \phi R+\frac{1}{2} \phi_{; \mu}^{\dagger} \phi^{; \mu}-V(\phi)\right] \sqrt{-g}+\mathcal{L}_{M} \sqrt{-g},
$$

where $\phi$ is an $U(N)$ iso-vector, $R$ the Ricci scalar, $\breve{\alpha}$ a dimensionless factor and $\mathcal{L}_{M}$ the Lagrange density of the fermionic and massless bosonic fields:

$$
\mathcal{L}_{M}=\frac{i}{2} \bar{\psi} \gamma_{L, R}^{\mu} \psi_{; \mu}+\text { h.c. }-\frac{1}{16 \pi} F_{\mu \nu}^{a} F_{a}^{\mu \nu}-k \bar{\psi}_{R} \phi^{\dagger} \hat{x} \psi_{L}+\text { h.c. }
$$

with the Yukawa coupling operator $\hat{x}$. Furthermore, there is a Higgs potential $\left(\mu^{2}<0, \lambda>0\right.$ real valued constants)

$$
V(\phi)=\frac{\mu^{2}}{2} \phi^{\dagger} \phi+\frac{\lambda}{24}\left(\phi^{\dagger} \phi\right)^{2}+\frac{3}{2} \frac{\mu^{4}}{\lambda} .
$$

The field equations for gravity and the Higgs field following from Eq.(3) are

$$
\begin{aligned}
R_{\mu \nu} & -\frac{1}{2} R g_{\mu \nu}+\frac{8 \pi}{\breve{\alpha} \phi^{\dagger} \phi} V(\phi) g_{\mu \nu}=-\frac{8 \pi}{\breve{\alpha} \phi^{\dagger} \phi} T_{\mu \nu}-\frac{4 \pi}{\breve{\alpha} \phi^{\dagger} \phi}\left[\phi_{; \mu}^{\dagger} \phi_{; \nu}+\phi_{; \nu}^{\dagger} \phi_{; \mu}\right]+ \\
& +\frac{4 \pi}{\breve{\alpha} \phi^{\dagger} \phi} \phi_{; \lambda}^{\dagger} \phi^{; \lambda} g_{\mu \nu}-\frac{1}{\phi^{\dagger} \phi}\left[\left(\phi^{\dagger} \phi\right)_{; \mu ; \nu}-\left(\phi^{\dagger} \phi\right)_{; \beta}^{; \beta} g_{\mu \nu}\right], \\
\phi_{; \mu}^{; \mu} & -\frac{1}{8 \pi} \breve{\alpha} \phi R+\mu^{2} \phi+\frac{\lambda}{6}\left(\phi^{\dagger} \phi\right) \phi=-2 k \bar{\psi}_{R} \hat{x} \psi_{L},
\end{aligned}
$$

where $T_{\mu \nu}$ is the energy-momentum tensor belonging to the matter Lagrangian $\mathcal{L}_{M}$ in Eq.(3). It satisfies the conservation law:

$$
T_{\mu}^{\nu}{ }_{; \nu}=k \bar{\psi}_{R} \phi_{; \mu}^{\dagger} \hat{x} \psi_{L}+\text { h.c. }+F_{\mu \nu}^{a} j_{a}^{\nu}(\phi) .
$$

$j_{a}^{\nu}(\phi)$ are the currents of the scalar field $\phi$. The coupling operator $\hat{x}$ is not zero in the case of a coupling of the scalar field with the fermionic field in the matter Lagrangian $\mathcal{L}_{M}$ in Eq.(4). Otherwise, it is zero. Instead of $\phi$ we go on to the real-valued function $\varphi$

$$
\varphi^{2}=\frac{\phi^{\dagger} \phi}{v^{2}}, \quad \text { with } v^{2}=\phi_{0}^{\dagger} \phi_{0}=-\frac{6 \mu^{2}}{\lambda}
$$

which describes this Higgs field normalized to the ground state $\phi_{0}=v N\left(N^{\dagger} N=1\right)$ of lowest energy. With the excited field $\xi$ following from

$$
\varphi^{2}=1+\xi
$$

and with

$$
G=\left(\breve{\alpha} v^{2}\right)^{-1}
$$

for the gravitational constant, and with the length scale

$$
l^{-2}=16 \pi G \frac{\mu^{4}}{\lambda}\left(1+\frac{4 \pi}{3 \breve{\alpha}}\right)^{-1}=-\frac{8 \pi}{3} \frac{\mu^{2}}{\breve{\alpha}}\left(1+\frac{4 \pi}{3 \breve{\alpha}}\right)^{-1},
$$

\footnotetext{
${ }_{;}^{4} \mu$ means the covariant derivative with respect to all gauged groups.

${ }^{5}$ The potential $V(\phi)$ is normalized to zero for the ground-state. Otherwise, a formal cosmological constant appears, whereas for Eq.5 only a cosmological function exists.

${ }^{6}$ In view of the structure of $l$ with the high values of $\breve{\alpha}$, only large values of the length scale $l$ are expected (c.f. [5] 6]). Further, for the extreme case $l \rightarrow \infty$, the symmetry can stay broken and the scalar field $\xi$ act antigravitationally in the exact solution of the statical field equations [3], avoiding, for instance, a Schwarzschild horizon.
} 
the field equations (6) and (7) with the use of $\breve{\alpha} \simeq\left(\frac{M_{\text {Planck }}}{M_{\text {Boson }}}\right)^{2} \gg 1$ (c.f. Eq.(11) become

$$
\begin{aligned}
& R_{\mu \nu}-\frac{1}{2} R g_{\mu \nu}+\frac{1}{l^{2}}(1+\xi)^{-1} \xi\left(1+\frac{3}{4} \xi\right) g_{\mu \nu}= \\
&=-8 \pi G(1+\xi)^{-1}\left(T_{\mu \nu}-\frac{\hat{q}}{3} T g_{\mu \nu}\right)-(1+\xi)^{-1} \xi_{, \mu ; \nu}, \\
& \xi_{; \mu}^{, \mu}+\frac{1}{l^{2}} \xi=\hat{q} \frac{8 \pi G}{3} T .
\end{aligned}
$$

$\hat{q}$ is an operator with the value 0 for a coupling of $\phi$ with the fermionic field through $\mathcal{L}_{M}(\hat{=} \hat{x} \neq 0)$ and with the value 1 for only coupling through the curvature scalar $R(\hat{=} \hat{x}=0)$. For the first case, the source of the scalar field vanishes identically [11].

In Eq.(13) there exists a cosmological function

$$
\Lambda(\xi)=\frac{8 \pi G}{1+\xi} V(\xi)=12 \pi G \frac{\mu^{4}}{\lambda} \frac{\xi^{2}}{1+\xi},
$$

which vanishes for the ground state $\xi=0$. This is a consequence of the special choice $V\left(\phi_{0}\right)=0$, according to Eq.(5) (see footnote 2).

In the field equation (13), it is possible to eliminate the Ricci scalar $R$ by multiplying Eq.(13) by $g_{\mu \nu}$. Now one obtains from Eq.(13) after the use of (14):

$$
\begin{aligned}
R_{\mu \nu}-\frac{1}{2 l^{2}} & (1+\xi)^{-1} \xi\left(1+\frac{3}{2} \xi\right) g_{\mu \nu}= \\
& =-8 \pi G(1+\xi)^{-1}\left[T_{\mu \nu}-\frac{1}{2}\left(1-\frac{\hat{q}}{3}\right) T g_{\mu \nu}\right]-(1+\xi)^{-1} \xi_{, \mu ; \nu}
\end{aligned}
$$

For calculating astrophysical problems, the metric for spherical symmetry

$$
d s^{2}=e^{\nu}\left(d x^{0}\right)^{2}-e^{\lambda} d r^{2}-r^{2}\left(d \vartheta^{2}+\sin ^{2} \vartheta d \varphi^{2}\right)
$$

is introduced, and the energy-momentum tensor $T_{\mu \nu}$ is taken phenomenologically as that of the ideal liquid ( $u^{\mu}:$ 4-velocity):

$$
T_{\mu \nu}=(\varrho+p) u_{\mu} u_{\nu}-p g_{\mu \nu}, \quad u^{\mu} u_{\mu}=1,
$$

with the pressure $p$ and density distribution $\varrho$. Hence, the field equations yield ( $u$ is the radial 3-velocity of the fluid):

$$
\begin{aligned}
& e^{\nu-\lambda}\left(\frac{\nu^{\prime \prime}}{2}+\frac{\nu^{\prime 2}}{4}-\frac{\nu^{\prime} \lambda^{\prime}}{4}+\frac{\nu^{\prime}}{r}\right)-\frac{\ddot{\lambda}}{2}-\frac{\dot{\lambda}^{2}}{4}+\frac{\dot{\lambda} \dot{\nu}}{4}+\frac{1}{2 l^{2}}(1+\xi)^{-1} \xi\left(1+\frac{3}{2} \xi\right) e^{\nu}= \\
& =8 \pi G(1+\xi)^{-1}\left[\left(e^{-\nu}-u^{2} e^{-\lambda}\right)^{-1}\left(\varrho+u^{2} p e^{\nu-\lambda}\right)-\frac{1}{2}\left(1-\frac{\hat{q}}{3}\right)(\varrho-3 p) e^{\nu}\right]+ \\
& \quad+(1+\xi)^{-1}\left[\ddot{\xi}-\frac{\dot{\nu}}{2} \dot{\xi}-\frac{\nu^{\prime}}{2} e^{\nu-\lambda} \xi^{\prime}\right], \\
& e^{\lambda-\nu}\left(\frac{\ddot{\lambda}}{2}+\frac{\dot{\lambda}^{2}}{4}-\frac{\dot{\lambda} \dot{\nu}}{4}\right)-\frac{\nu^{\prime \prime}}{2}-\frac{\nu^{\prime 2}}{4}+\frac{\nu^{\prime} \lambda^{\prime}}{4}+\frac{\lambda^{\prime}}{r}-\frac{1}{2 l^{2}}(1+\xi)^{-1} \xi\left(1+\frac{3}{2} \xi\right) e^{\lambda}= \\
& =8 \pi G(1+\xi)^{-1}\left[\left(e^{-\nu}-u^{2} e^{-\lambda}\right)^{-1}\left(u^{2} \varrho+p e^{\lambda-\nu}\right)+\frac{1}{2}\left(1-\frac{\hat{q}}{3}\right)(\varrho-3 p) e^{\lambda}\right]+ \\
& +(1+\xi)^{-1}\left[\xi^{\prime \prime}-\frac{\dot{\lambda}}{2} e^{\lambda-\nu} \dot{\xi}-\frac{\lambda^{\prime}}{2} \xi^{\prime}\right]
\end{aligned}
$$




$$
\begin{aligned}
\frac{\dot{\lambda}}{r} & =-8 \pi G(1+\xi)^{-1}\left[e^{-\nu}-u^{2} e^{-\lambda}\right]^{-1}(p+\varrho) u-(1+\xi)^{-1}\left[\dot{\xi}^{\prime}-\frac{\nu^{\prime}}{2} \dot{\xi}-\frac{\dot{\lambda}}{2} \xi^{\prime}\right], \\
e^{-\lambda} & \left(1+\frac{r}{2}\left(\nu^{\prime}-\lambda^{\prime}\right)\right)-1+\frac{r^{2}}{2 l^{2}}(1+\xi)^{-1} \xi\left(1+\frac{3}{2} \xi\right)= \\
& =-8 \pi G(1+\xi)^{-1}\left[p r^{2}+\frac{1}{2}\left(1-\frac{\hat{q}}{3}\right)(\varrho-3 p) r^{2}\right]-(1+\xi)^{-1} r e^{-\lambda} \xi^{\prime},
\end{aligned}
$$

with the Higgs equation for the excited Higgs field:

$$
\ddot{\xi} e^{-\nu}-\xi^{\prime \prime} e^{-\lambda}-\frac{\dot{\nu}-\dot{\lambda}}{2} e^{-\nu} \dot{\xi}-\frac{\nu^{\prime}-\lambda^{\prime}}{2} e^{-\lambda} \xi^{\prime}-\frac{2}{r} e^{-\lambda} \xi^{\prime}+\frac{1}{l^{2}} \xi=-\hat{q} \frac{8 \pi G}{3}(\varrho-3 p) .
$$

For the following, we take $\hat{q}=1$. The other case can be found in Dehnen and Frommert [11]. There, Higgs particles only interact through the gravitational channel.

\section{The Linearized Field Equations}

We will examine the field equations for the case $\hat{q}=1$, i.e., the case of no coupling of $\phi$ through $\mathcal{L}_{M}$. Therefore, the source of this Higgs field does not disappear and the Higgs particles are able to be generated, in contrast to the case of a coupling through $\mathcal{L}_{M}$ with only gravitationally coupled Higgs particles [11]. Furthermore, for the investigation of the flat rotation curves of galaxies only the knowledge of the functions $\nu(r)$ and $\xi(r)$ is necessary. For them we find in static linear approximation, from Eqs.(19)-(23) the field equations for $\nu$ and $\xi$ :

$$
\begin{aligned}
\left(\frac{1}{2} \nu^{\prime \prime}+\frac{1}{r} \nu^{\prime}\right)+\frac{1}{2 l^{2}} \xi & =8 \pi G\left(\frac{2}{3} \varrho+p\right), \\
\xi^{\prime \prime}+\frac{2}{r} \xi^{\prime}-\frac{1}{l^{2}} \xi & =-\frac{8 \pi G}{3}(\varrho-3 p) .
\end{aligned}
$$

However, in the case of a singular central mass, a non-linearization of the $\xi$-field is required for the central area because the effective gravitational coupling parameter $G(\xi) \sim(1+\xi)^{-1}$ in Eqs.(19)-(22) runs to zero for $r \rightarrow 0$ (see Eq.(47)). For this reason, it is possible to linearize the field equations in $\nu$ and $\lambda$ but not in $\xi$ in the centre. This partially linearized equation for $\nu(r)$ can be written instead of (24) in the form (c.f. Eq.(19))

$$
\frac{\nu^{\prime \prime}}{2}+\frac{1}{r} \nu^{\prime}+\frac{1}{2 l^{2}} \xi \frac{1+\frac{3}{2} \xi}{1+\xi}=8 \pi G \frac{\frac{2}{3} \varrho+p}{1+\xi} .
$$

In contrast to this, the $\xi$-field equation (25) is valid without any change (c.f. Eq.(23)).

On the basis of Eqs.(24)-(25) we analyze in the following the rotation curves of galaxies. Of course, besides these, further investigations within this theory are necessary concerning, for instance, the gravitational lensing effect and the Tully-Fisher law for galaxies.

\section{Sphere-like galaxy with a singular mass in the centre}

For simplicity, we choose a mass-sphere (radius $R_{0}$ ) with a central point mass as galaxy model. The pressure of the sphere is given by a polytropic equation of state and that of the central mass $\varrho_{S}=M_{S} \delta(\vec{x})$ may be given by an extreme equation of state

$$
p_{S}=a \varrho_{S}=a M_{S} \delta(\vec{x})
$$


with $a$ as the pressure parameter $\left(0<a \leq 1\right.$, but $\left.a \neq \frac{1}{3}\right) 7$; $M_{S}$ is the central mass.

We solve the equations (24) up to (26) in several steps:

- The $\xi$-field for $r>R_{0}$ :

Outside of the sphere $\left(r>R_{0}\right), p=\varrho=0$ is valid. The general outer solution for the $\xi$-field with the boundary condition to be zero in infinity $(r \rightarrow \infty)$ is given through

$$
\xi_{a}=\frac{A}{r} e^{-r / l}
$$

with the integration constant $A$.

- The potential of the gravitational field for $r>R_{0}$ :

Multiplying Eq.(26) for the vacuum with $2 r^{2}$ gives

$$
\left(r^{2} \nu^{\prime}\right)^{\prime}+\frac{r^{2}}{l^{2}} \xi \frac{1+\frac{3}{2} \xi}{1+\xi}=0 .
$$

The quotient $\left(1+\frac{3}{2} \xi\right) /(1+\xi)$ has the value $\frac{3}{2}$ for $r \rightarrow 0$ and 1 for $r \rightarrow \infty$ and is everywhere monotonous. For this reason, it may be possible to approximate it by one. Therefore, it follows according to Eq.(28):

$$
\left(r^{2} \nu_{a}^{\prime}\right)^{\prime}+\frac{1}{l^{2}} A r e^{-r / l}=0
$$

After integration one finds

$$
\nu_{a}^{\prime}=\frac{A}{r^{2}} e^{-r / l}\left(1+\frac{r}{l}\right)+\frac{B}{r^{2}}
$$

with $B$ as an integration constant.

The integration of Eq. (31) yields

$$
\nu_{a}=-\frac{A}{r} e^{-r / l}-\frac{B}{r}
$$

using the boundary condition to be zero in infinity. The specific form of the integration constants $A$ and $B$ depends on the density distribution of the galaxy, i.e., on the inner solution of the field equations, according to the continuity conditions at the surface $r=R_{0}$.

- The fields for $r \leq R_{0}$ and the boundary and continuity conditions:

In the case $\hat{U}=1$ the energy-momentum law (18) is the same as in General Relativity. Octavio Obregón calculated in 1971 the general relativistic barometric formulae of matter in the case of hydrostatic equilibrium and found for the coupling of pressure and density of polytropic systems with the equation of state $p=\alpha \varrho^{\gamma}$ (polytropic amplitude $\alpha$ ), the density distribution [12]

$$
\varrho=\left(\frac{1}{\alpha}\right)^{\frac{1}{\gamma-1}}\left[\left(\frac{\zeta}{\zeta_{s}}\right)^{\frac{\gamma-1}{\gamma}}-1\right]^{\frac{1}{\gamma-1}}
$$

\footnotetext{
${ }^{7}$ The case $a=\frac{1}{3}$ must be considered separately, which is not performed in this paper.
} 
with $\zeta=e^{\nu / 2}$ as the absolute value of the time-like Killing vector and $\zeta_{s}=e^{\nu_{s} / 2}$ as its value at the surface of the distribution of matter. For $|\nu| \ll 1$, the density distribution reads

$$
\varrho=\left(\frac{1}{\alpha}\right)^{\frac{1}{\gamma-1}}\left[\frac{1}{2} \frac{\gamma-1}{\gamma}\left(\nu_{s}-\nu\right)\right]^{\frac{1}{\gamma-1}} .
$$

For $\gamma=2$, the differential equations (24)-(26) become linear in $\nu$ concerning $\varrho$, in accordance with the linearized Einstein theory. Then, the polytropic equation becomes

$$
p=\alpha \varrho^{2}, \quad \varrho=\frac{1}{4 \alpha}\left(\nu_{s}-\nu\right) .
$$

Therefore, $p$ can then be neglected in Eqs.(24), (25) and (26) in the linear approximation. The pressure parameter $a$ and the polytropic amplitude $\alpha$ can be fitted differently from galaxy to galaxy as well as the central mass $M_{S}$ and the mass $M$ of the sphere or its radius $R_{0}$. The fundamental length $l$, however, should have the same value for all galaxies. This value may further be constrained from an analysis within the Friedmann-Lemaître cosmology with Robertson-Walker metric, too.

Of course, instead of with the polytropic equation of state one might start also with the empirical mass distribution according to the surface brightness of the galaxies. However, then the rotation of the galaxies would have to be taken into account in order to avoid unphysical equations of state, and the mass-to-light ratio must be investigated in our scalar-tensor theory. This will be performed in a further paper.

The exterior field components $\left(r>R_{0}\right)$ are given through Eqs.(28) and (32). For the inner field $\left(r \leq R_{0}\right)$, there is, according to (24), (25) and (35):

$$
\begin{aligned}
\nu_{i}= & {\left[\nu_{s}-\frac{1}{r}\left(\tilde{A} \sin \left(k \frac{r}{l}\right)+\tilde{B} \cos \left(k \frac{r}{l}\right)+\tilde{C} \sinh \left(\kappa \frac{r}{l}\right)+\tilde{D} \cosh \left(\kappa \frac{r}{l}\right)\right)\right], } \\
\xi_{i}= & \frac{1}{r}\left[\tilde{A}\left(\delta-k^{2}\right) \sin \left(k \frac{r}{l}\right)+\tilde{B}\left(\delta-k^{2}\right) \cos \left(k \frac{r}{l}\right)+\right. \\
& \left.+\tilde{D}\left(\delta+\kappa^{2}\right) \cosh \left(\kappa \frac{r}{l}\right)+\tilde{C}\left(\delta+\kappa^{2}\right) \sinh \left(\kappa \frac{r}{l}\right)\right]
\end{aligned}
$$

with the definitions

$$
\begin{aligned}
& k^{2}=\frac{1}{2}\left[\delta-1+\sqrt{\delta^{2}+\delta+1}\right. \\
& \kappa^{2}=\frac{1}{2}\left[1-\delta+\sqrt{\delta^{2}+\delta+1}\right]
\end{aligned}
$$

and

$$
\frac{\delta}{l^{2}}=\frac{8 \pi G}{3 \alpha}
$$

$\tilde{A}, \tilde{B}, \tilde{C}$ and $\tilde{D}$ are integration constants, essentially defined through the behaviour at $r=0$ and the continuity conditions at the surface $r=R_{0}$. Because in Eq.(26) the effective gravitational coupling parameter $G(\xi)=G /(1+\xi)$ runs to zero for $r \rightarrow 0$ in the case of a central singular mass $M_{S}$ (c.f. (47)), this singular mass has no direct influence on the behaviour of $\nu$. It is only active on $\xi$, according to Eqs.(23) or (25) (see Eqs.(47) and (51)), and herewith only indirectly on $\nu$ with respect to (26). However, the singular behaviour of $\xi$ for $r \rightarrow 0$ (see Eq.(47)) implies according to Eq.(26) no singular behaviour of $\nu$ for $r \rightarrow 0$. Therefore, $\nu$ must be regular for $r \rightarrow 0$. So, it follows from (36) immediately

$$
\tilde{D}=-\tilde{B}
$$


The continuity conditions for $\nu$ and $\xi$ and their derivatives with respect to $r$ at $r=R_{0}$ lead to

$$
\begin{gathered}
\tilde{A} \sin \left(k \frac{R_{0}}{l}\right)+\tilde{B}\left[\cos \left(k \frac{R_{0}}{l}\right)-\cosh \left(\kappa \frac{R_{0}}{l}\right)\right]+\tilde{C} \sinh \left(\kappa \frac{R_{0}}{l}\right)=0 \\
\left\{\tilde{A} k \cos \left(k \frac{R_{0}}{l}\right)-\tilde{B}\left[k \sin \left(k \frac{R_{0}}{l}\right)+\kappa \sinh \left(\kappa \frac{R_{0}}{l}\right)\right]+\tilde{C} \kappa \cosh \left(\kappa \frac{R_{0}}{l}\right)\right\}=l \nu_{s}-A e^{-R_{0} / l} \\
\left\{\tilde{A} k^{2} \sin \left(k \frac{R_{0}}{l}\right)+\tilde{B}\left[k^{2} \cos \left(k \frac{R_{0}}{l}\right)+\kappa^{2} \cosh \left(\kappa \frac{R_{0}}{l}\right)\right]-\tilde{C} \kappa^{2} \sinh \left(\kappa \frac{R_{0}}{l}\right)\right\}=-A e^{-R_{0} / l} \\
\left\{\tilde{A} k^{3} \cos \left(k \frac{R_{0}}{l}\right)-\tilde{B}\left[k^{3} \sin \left(k \frac{R_{0}}{l}\right)-\kappa^{3} \sinh \left(\kappa \frac{R_{0}}{l}\right)\right]-\tilde{C} \kappa^{3} \cosh \left(\kappa \frac{R_{0}}{l}\right)\right\}= \\
=\delta l \nu_{s}+A(1-\delta) e^{-R_{0} / l}
\end{gathered}
$$

where $\nu_{s}$ is given by (see Eq.(32)):

$$
\nu_{s}=-\frac{A}{R_{0}} e^{-R_{0} / l}-\frac{B}{R_{0}} .
$$

From Eq.41) it follows immediately

$$
\tilde{A}=\tilde{B} \frac{\cosh \left(\kappa \frac{R_{0}}{l}\right)-\cos \left(k \frac{R_{0}}{l}\right)}{\sin \left(k \frac{R_{0}}{l}\right)}-\tilde{C} \frac{\sinh \left(\kappa \frac{R_{0}}{l}\right)}{\sin \left(k \frac{R_{0}}{l}\right)} .
$$

On the other hand, the $\xi_{i}$ field becomes for $r \rightarrow 0$, according to Eq. 37):

$$
\xi_{i} \rightarrow-\frac{\tilde{B}}{r}\left(k^{2}+\kappa^{2}\right) .
$$

The integration constant $\tilde{B}$ can be coupled to the central mass, using the equation of state (27) for the centre of the polytropic sphere; equation (25) can then be rewritten:

$$
\Delta \xi-\frac{1}{l^{2}} \xi=-\frac{8 \pi G}{3}\left[M_{S} \delta(\vec{x})(1-3 a)+\varrho\right]
$$

with the pressure parameter $a$ and central mass $M_{S}$. The connection between $\tilde{B}$ and the singular mass $M_{S}$ is found through integration of Eq.(48) for the sphere of radius $r$ and then taking the limit $r \rightarrow 0$. In

$$
\lim _{r \rightarrow 0} \int_{V}\left(\Delta \xi-\frac{1}{l^{2}} \xi+\frac{8 \pi G}{3} \varrho\right) d V
$$

the term $\lim _{r \rightarrow 0} \int_{V}\left(\frac{1}{l^{2}} \xi-\frac{8 \pi G}{3} \varrho\right) d V$ does not contribute, since the integral goes to zero as $r^{2}$. The volume integral of $\Delta \xi$ can be transformed with the help of the Gauss theorem to a surface integral. Hence, with the solution (47), we get from Eq.(48):

$$
\begin{aligned}
\lim _{r \rightarrow 0} \int_{V} \Delta \xi d V & =\lim _{r \rightarrow 0} \oint_{F} \nabla \xi d f= \\
& =4 \pi \tilde{B}\left(k^{2}+\kappa^{2}\right)=-\frac{8 \pi G}{3} M_{s}(1-3 a) .
\end{aligned}
$$

Thus, we have

$$
\tilde{B}=-\frac{2}{3} G M_{S} \frac{1-3 a}{k^{2}+\kappa^{2}}
$$


Furthermore, an expression for the mass of the polytropic sphere is necessary. From Eqs.(35) and (36), it follows:

$$
\begin{gathered}
M=\frac{\pi}{\alpha} \int_{0}^{R}\left(\nu_{s}-\nu\right) r^{2} d r=\frac{\pi}{\alpha}\left[\tilde{A}\left(\left(\frac{l}{k}\right)^{2} \sin \left(k \frac{R_{0}}{l}\right)-R_{0} \frac{l}{k} \cos \left(k \frac{R_{0}}{l}\right)\right)+\right. \\
\quad+\tilde{B}\left(\left(\frac{l}{k}\right)^{2} \cos \left(k \frac{R_{0}}{l}\right)+R_{0} \frac{l}{k} \sin \left(k \frac{R_{0}}{l}\right)-\left(\frac{l}{k}\right)^{2}\right)+\tilde{C}\left(R_{0} \frac{l}{\kappa} \cosh \left(\kappa \frac{R_{0}}{l}\right)-\right. \\
\left.\left.\quad-\left(\frac{l}{\kappa}\right)^{2} \sinh \left(\kappa \frac{R_{0}}{l}\right)\right)-\tilde{B}\left(\frac{R_{0} \kappa}{l}-\left(\frac{l}{\kappa}\right)^{2} \cosh \left(\kappa \frac{R_{0}}{l}\right)+\left(\frac{l}{\kappa}\right)^{2}\right)\right]
\end{gathered}
$$

Herewith, all integration constants are determined. They can be put into Eq.(36) and (37) and in the vacuum solutions (28) and (32).

The four equations (41)-(44) and the two equations (51) and (52) determine the parameters $\tilde{A}, \tilde{B}$, $\tilde{C}, A, B$ and $R_{0}$ in dependence of the variable masses $M$ and $M_{S}$; the quantities $a, \alpha$ and $l$ are to be considered as fundamental natural constants, the values of which are unknown of course, and which can be defined in a suitable way. Obviously, there exists in general a mass-radius relation $R_{0}\left(M, M_{S}\right)$.

The resolution of Eqs.(42)-(46) gives the following results: $\tilde{B}$ is determined immediately, according to Eq.(51) by the value of $M_{S}$. For the other coefficients it follows then successively, for $A$ :

$$
\begin{aligned}
\frac{A e^{-R_{0} / l}}{k^{2}+\kappa^{2}}[ & \left.k^{2}+\kappa^{2}+\left(k^{3}-\delta k\right) \cot \left(k \frac{R_{0}}{l}\right)+\left(\kappa^{3}+\delta \kappa\right) \operatorname{coth}\left(\kappa \frac{R_{0}}{l}\right)\right] \\
= & \tilde{B}\left[\left(\kappa^{3}+\delta \kappa\right) \sinh \left(\kappa \frac{R_{0}}{l}\right)\left(1-\operatorname{coth}^{2}\left(\kappa \frac{R_{0}}{l}\right)\right)-\right. \\
& \left.-\left(k^{3}-\delta k\right) \sin \left(k \frac{R_{0}}{l}\right)\left(1+\cot ^{2}\left(k \frac{R_{0}}{l}\right)\right)\right] .
\end{aligned}
$$

Then, for $B$ :

$$
\begin{aligned}
\frac{B l}{R_{0}}= & A\left[\frac{e^{-R_{0} / l}}{k^{2}+\kappa^{2}}\left(k \cot \left(k \frac{R_{0}}{l}\right)-\kappa \operatorname{coth}\left(\kappa \frac{R_{0}}{l}\right)\right)-\left(1+\frac{l}{R_{0}}\right) e^{-R_{0} / l}\right]+ \\
& +\tilde{B}\left[k \sin \left(k \frac{R_{0}}{l}\right)+\kappa \sinh \left(\kappa \frac{R_{0}}{l}\right)+k\left(\cos \left(k \frac{R_{0}}{l}\right)-\cosh \left(\kappa \frac{R_{0}}{l}\right)\right) \cot \left(k \frac{R_{0}}{l}\right)+\right. \\
& \left.+\cosh \left(\kappa \frac{R_{0}}{l}\right)\left(k \cot \left(k \frac{R_{0}}{l}\right)-\kappa \operatorname{coth}\left(\kappa \frac{R_{0}}{l}\right)\right)\right]
\end{aligned}
$$

Finally:

$$
\begin{aligned}
& \tilde{A}=-\frac{A e^{-R_{0} / l}}{k^{2}+\kappa^{2}} \csc \left(k \frac{R_{0}}{l}\right)-\tilde{B} \cot \left(k \frac{R_{0}}{l}\right), \\
& \tilde{C}=\frac{A e^{-R_{0} / l}}{k^{2}+\kappa^{2}} \operatorname{csch}\left(\kappa \frac{R_{0}}{l}\right)+\tilde{B} \operatorname{coth}\left(\kappa \frac{R_{0}}{l}\right) .
\end{aligned}
$$

The value of $R_{0}$ is finally determined by Eq.(52) through the mass $M$ of the polytropic sphere, so that a mass-radius relation exists. However, this procedure is correct so long as $B \neq 0$, i.e. $M_{S} \neq 0$ (see Eq. (51)).

A totally different situation exists in the case of $\tilde{B}=0$, i.e. $M_{S}=0$. Then it follows from Eq. (53):

$$
k^{2}+\kappa^{2}+\left(k^{3}-\delta k\right) \cot \left(k \frac{R_{0}}{l}\right)+\left(\kappa^{3}+\delta \kappa\right) \operatorname{coth}\left(\kappa \frac{R_{0}}{l}\right)=0 .
$$


By this equation the radius $R_{0}$ is determined independently from the mass $M$ only by the natural parameters $\alpha, l$ and $G .8$ Furthermore, one finds from Eqs.(53)-(56):

$$
\begin{aligned}
\frac{B l}{R_{0}} & =A\left[\frac{e^{-R_{0} / l}}{k^{2}+\kappa^{2}}\left(k \cot \left(k \frac{R_{0}}{l}\right)-\kappa \operatorname{coth}\left(\kappa \frac{R_{0}}{l}\right)\right)-\left(1+\frac{l}{R_{0}}\right) e^{-R_{0} / l}\right], \\
\tilde{A} & =-\frac{A e^{-R_{0} / l}}{k^{2}+\kappa^{2}} \csc \left(k \frac{R_{0}}{l}\right), \\
\tilde{C} & =\frac{A e^{-R_{0} / l}}{k^{2}+\kappa^{2}} \operatorname{csch}\left(\kappa \frac{R_{0}}{l}\right) .
\end{aligned}
$$

The value of $A$ is then finally determined by the mass $M$, according to Eq.(52)

\section{Tangential velocity and Rotation curves}

For the tangential velocity $v_{t a n}=v^{\varphi}$ for central symmetry, it is valid [20]

$$
v_{\text {tan }}^{2} \equiv v^{2}=\frac{r \nu^{\prime}}{2} .
$$

Accordingly, the square velocity (61) is:

$$
\begin{aligned}
& \text { Inner region }\left(r \leq R_{0}\right) \text { : } \\
& \begin{aligned}
v_{i}^{2} & =\frac{1}{2 r}\left[\left(\tilde{B}-\tilde{A} \frac{k}{l} r\right) \cos \left(\frac{k r}{l}\right)-\left(\tilde{B}+\tilde{C} \frac{\kappa}{l} r\right) \cosh \left(\frac{\kappa r}{l}\right)+\right. \\
& \left.+\left(\tilde{A}+\tilde{B} \frac{k}{l} r\right) \sin \left(\frac{k r}{l}\right)+\left(\tilde{C}+\tilde{B} \frac{\kappa}{l} r\right) \sinh \left(\frac{\kappa r}{l}\right)\right] .
\end{aligned}
\end{aligned}
$$

Outer region $\left(r>R_{0}\right)$ :

$$
v_{a}^{2}=\frac{e^{-r / l}}{2 r}\left[A\left(1+\frac{r}{l}\right)+B e^{r / l}\right] \text {. }
$$

The integration constants $A, B, \tilde{A}, \tilde{B}$ and $\tilde{C}$ are given above (c.f. Eqs.(52) and (53)-(56)). For them, the gravitational coupling constant is $G=\frac{3}{4} G_{N}$ with $G_{N}$ as the Newtonian gravitational constant, because according to this theory, the determination of the gravitational constant in the laboratory [14] is performed under the condition $l \gg$ diameter of the torsion balance.

An estimation of the polytropic amplitude with the use of the caloric equation of state and the virial theorem leads to an approximate value:

$$
\alpha \approx \frac{G_{N} R_{0}^{2}}{c^{4}}, \quad \frac{\delta}{c^{4}} \approx\left(\frac{l}{R_{0}}\right)^{2} .
$$

With the Milky Way radius $R_{1}=2.5 \mathrm{kpc}$, it follows $\alpha \approx 2.5 \cdot 10^{29} \frac{\mathrm{s}^{2} \mathrm{~m}}{\mathrm{~kg}} / \mathrm{c}^{4}$, which is of the right order of magnitude according to the figures below.

The best fits for rotation curves of some galaxies are, in case of a non-vanishing central mass $M_{S}$ are found under figures 1 through 6.

In case of a vanishing central mass, i.e. $M_{S}=0$, the rotation curves for the same galaxies are found under figures 7 through 12.

${ }^{8}$ The same situation is realized in Newton's theory in case of the polytropic index 2 . 


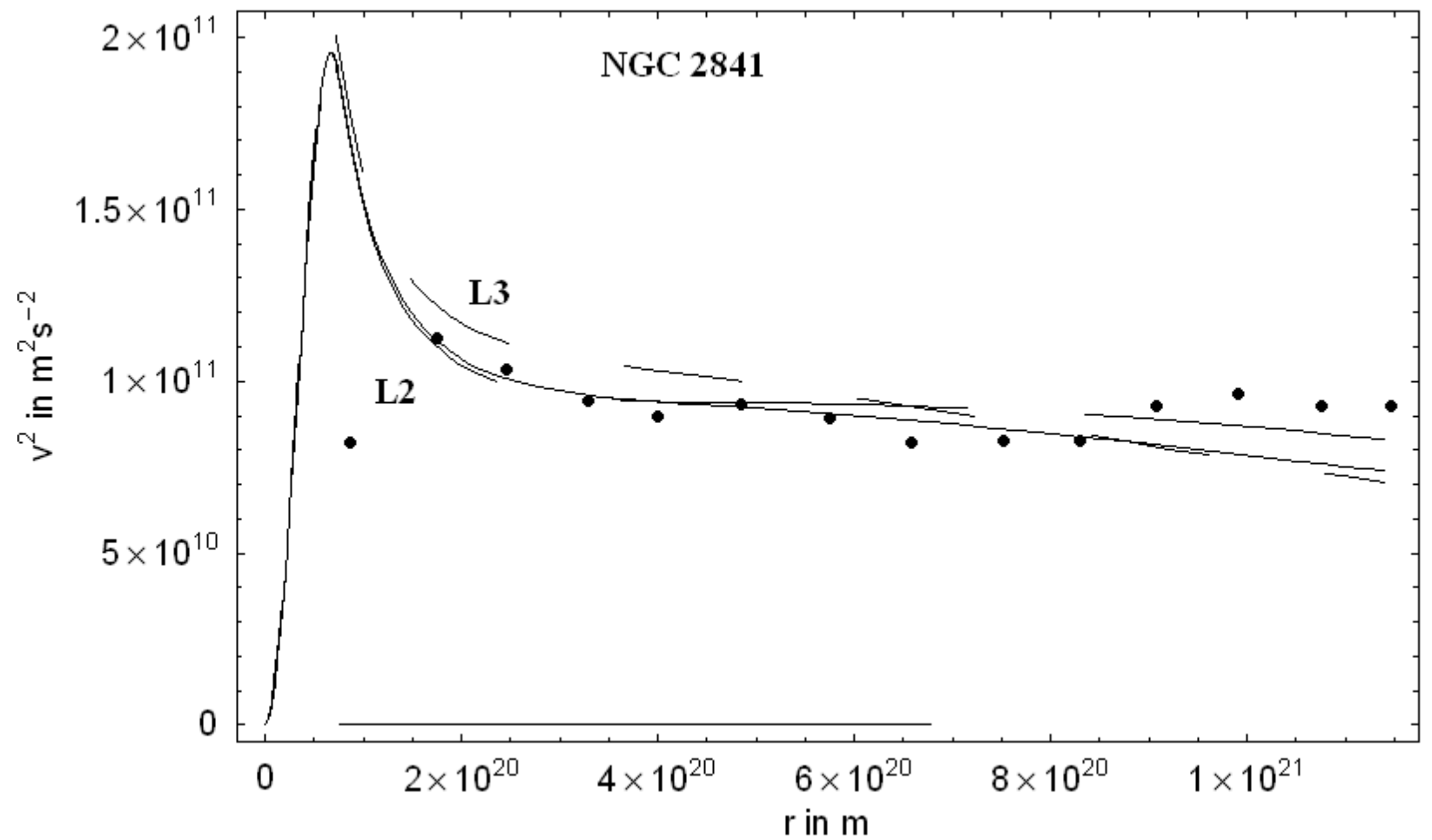

1: Rotation curves with a length scale $l=6 R_{1}$ (L2), $l=5 R_{1}$ (L1) and $l=4 R_{1}$ (L3) with experimental points for the galaxy NGC 2841 with $R_{0}=2.45 \mathrm{kpc}$ [24]. The galaxy mass is given as $M=8.5 \cdot 10^{10} M_{\text {sun }}$, with a central mass $M_{S} / M=180,140$ and 120, respectively, whereas the polytropic amplitude varies slowly according to $\alpha \cdot 10^{-30} \approx 0.27$, 0.27 and $0.28 \frac{s^{2} m}{k g} / c^{4}$, respectively. $R_{1}$ is the radius of the Milky Way.

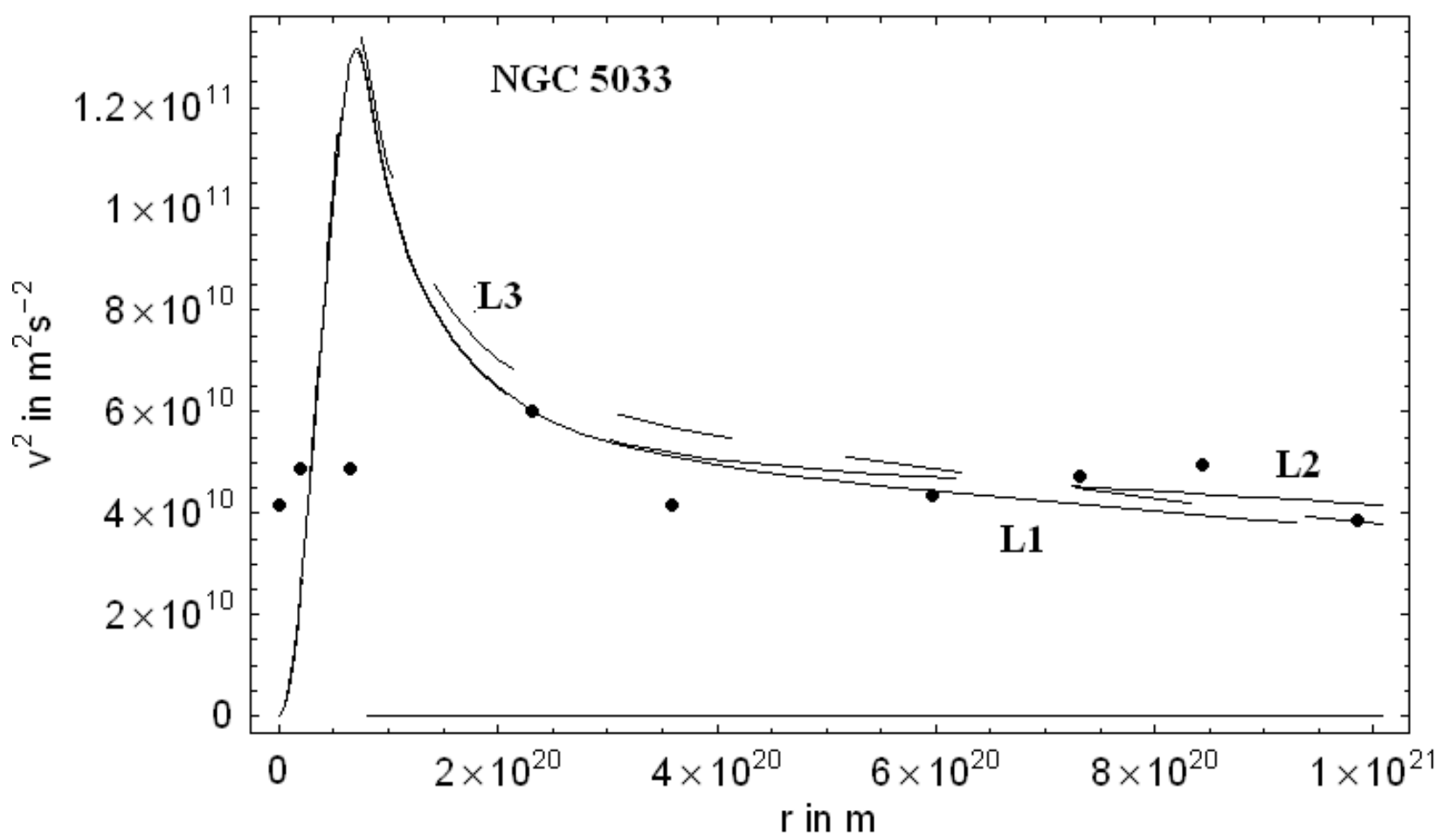

2: Rotation curves with a length scale as in Figure 1 and experimental points for the galaxy NGC 5033 [25]. There is $M=7 \cdot 10^{10} M_{\text {sun }}$. The galaxy radius is $R_{0}=2.6 \mathrm{kpc}$. The central mass is chosen as $M_{S} / M=80,60$ and 55, respectively, whereas the polytropic amplitude varies slowly according to $\alpha \cdot 10^{-30} \approx 0.29,0.29$ and $0.30 \frac{\mathrm{s}^{2} \mathrm{~m}}{\mathrm{~kg}} / \mathrm{c}^{4}$, respectively. 


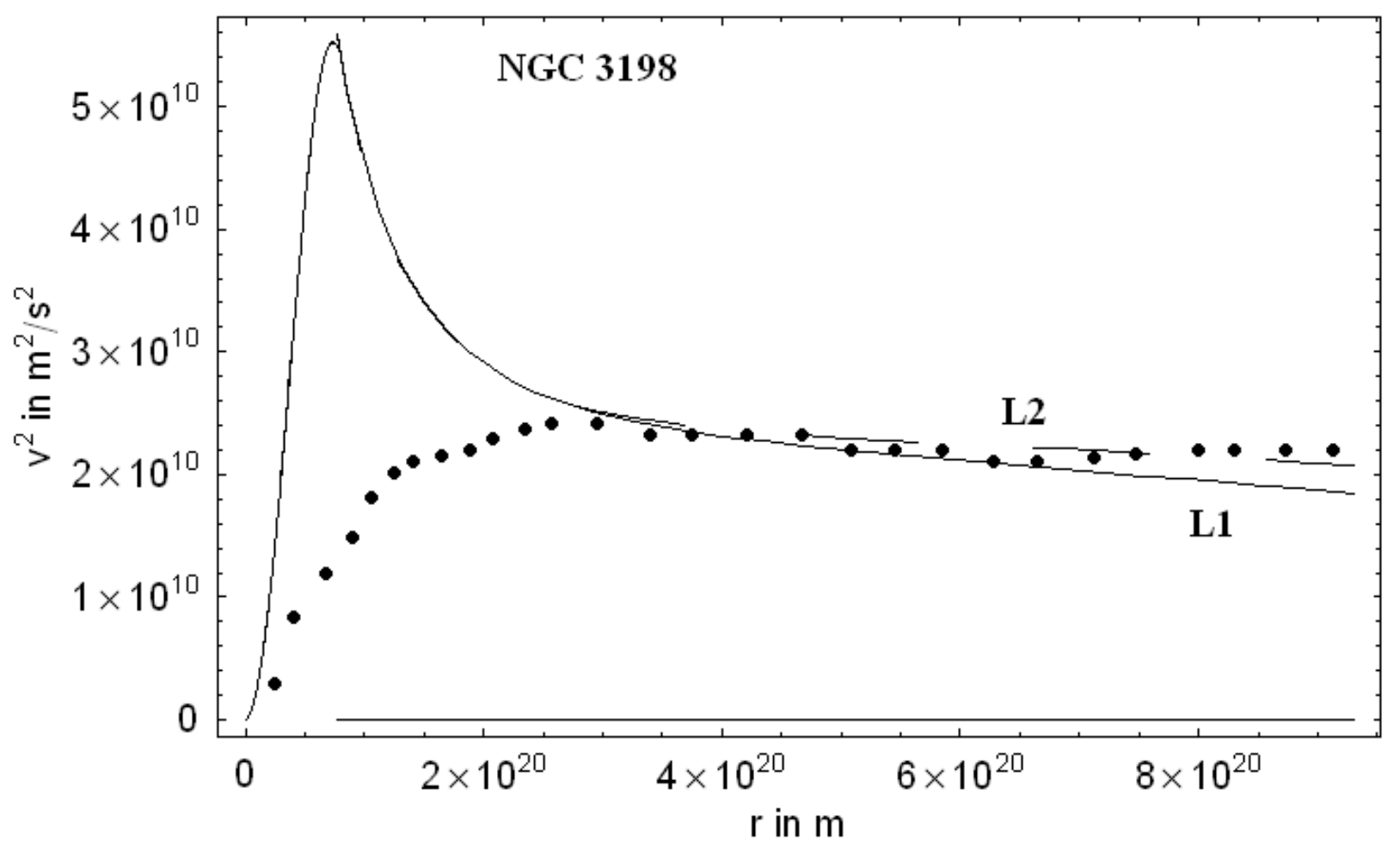

3: Rotation curves with a length scale as in Figure 1 and experimental points for the galaxy NGC 3198 with $R_{0}=2.68 \mathrm{kpc}$ [24]. The galaxy mass is taken as $M=3 \cdot 10^{10} M_{\text {sun }}$ and the central mass $M_{S} / M=40$, and 30, respectively, whereas the polytropic amplitude is $\alpha \cdot 10^{-30} \approx 0.32 \frac{\mathrm{s}^{2} m}{\mathrm{~kg}} / \mathrm{c}^{4}$.

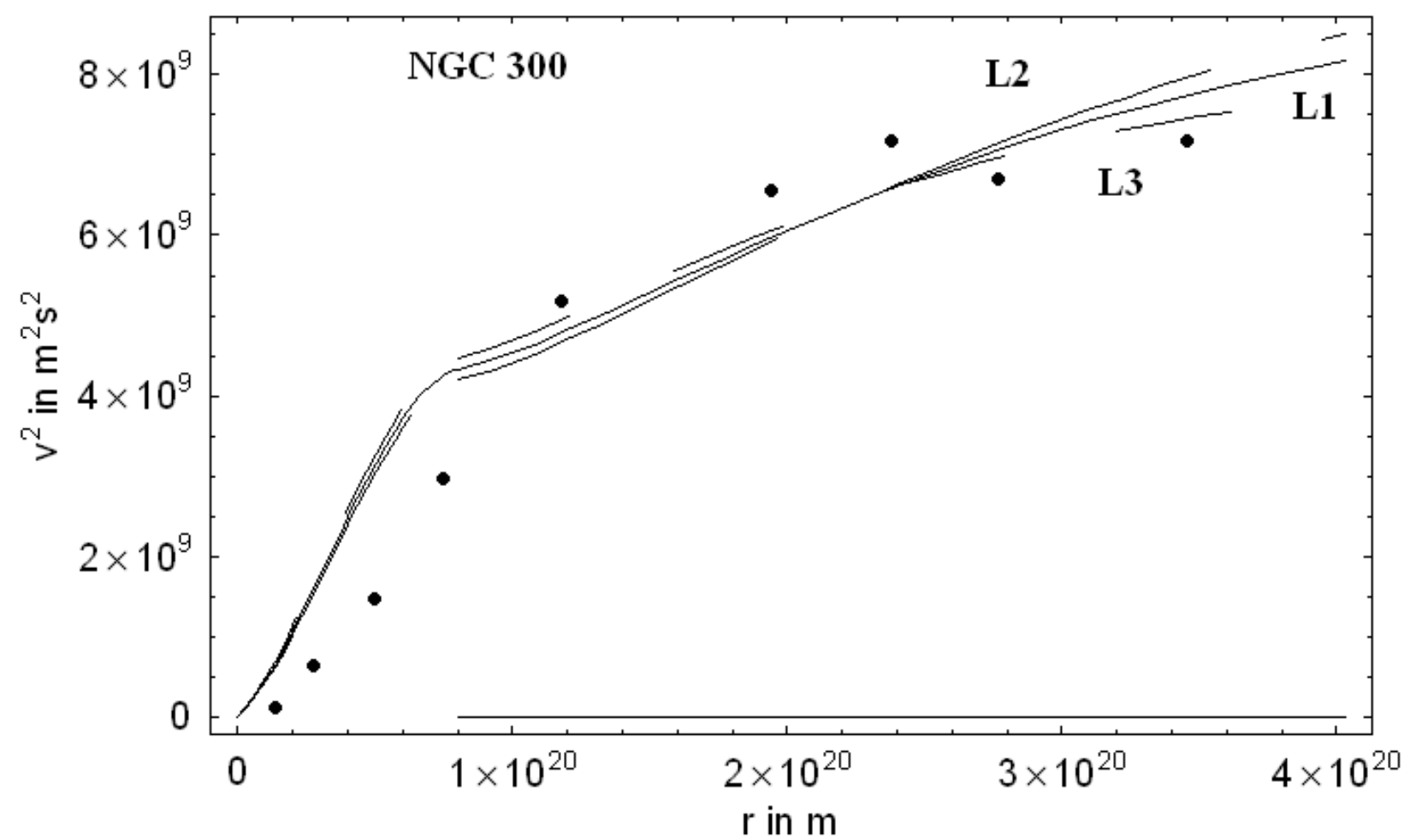

4: Rotation curves with a length scale as in Figure 1 and experimental points for the galaxy NGC 300 with $R_{0}=2.6 \mathrm{kpc}$ [24]. There is $M=0.1 \cdot 10^{10} M_{\text {sun }}$ and $M_{S} / M=23,17$ and 12 , respectively, whereas the polytropic amplitude varies slowly according to $\alpha \cdot 10^{-30} \approx 0.63,0.64$ and $0.67 \frac{\mathrm{s}^{2} \mathrm{~m}}{\mathrm{~kg}} / \mathrm{c}^{4}$, respectively. 


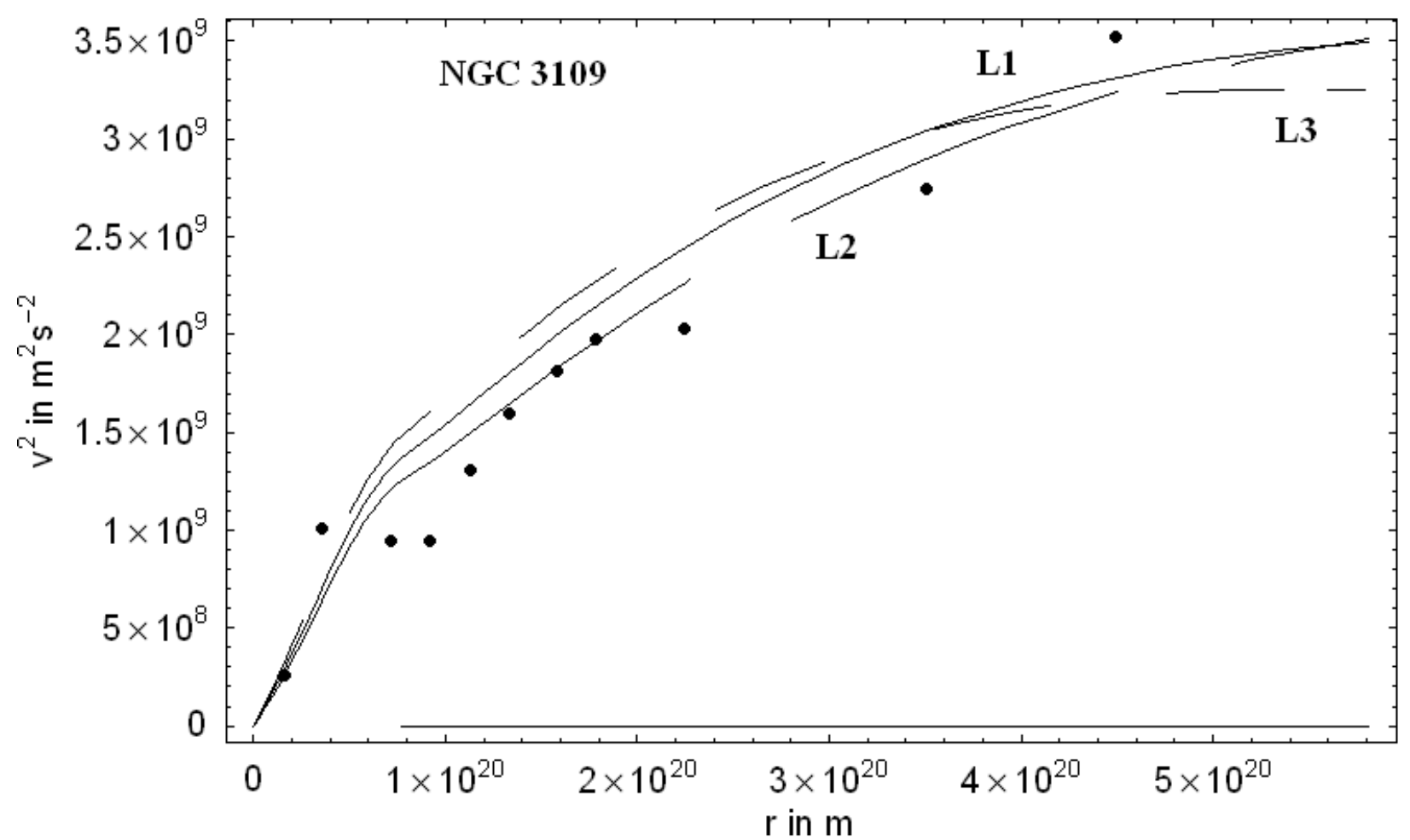

5: Rotation curves with a length scale as in Figure 1 and experimental points for the galaxy NGC 3109 with $R_{0}=2.5 \mathrm{kpc}$ [24]. There is $M=0.02 \cdot 10^{10} M_{\text {sun }}$ and $M_{S} / M=8.5,6.8$ and 5, respectively, whereas the polytropic amplitude varies slowly according to $\alpha \cdot 10^{-30} \approx 0.82,0.89$ and $0.97 \frac{\mathrm{s}^{2} \mathrm{~m}}{\mathrm{~kg}} / \mathrm{c}^{4}$, respectively.

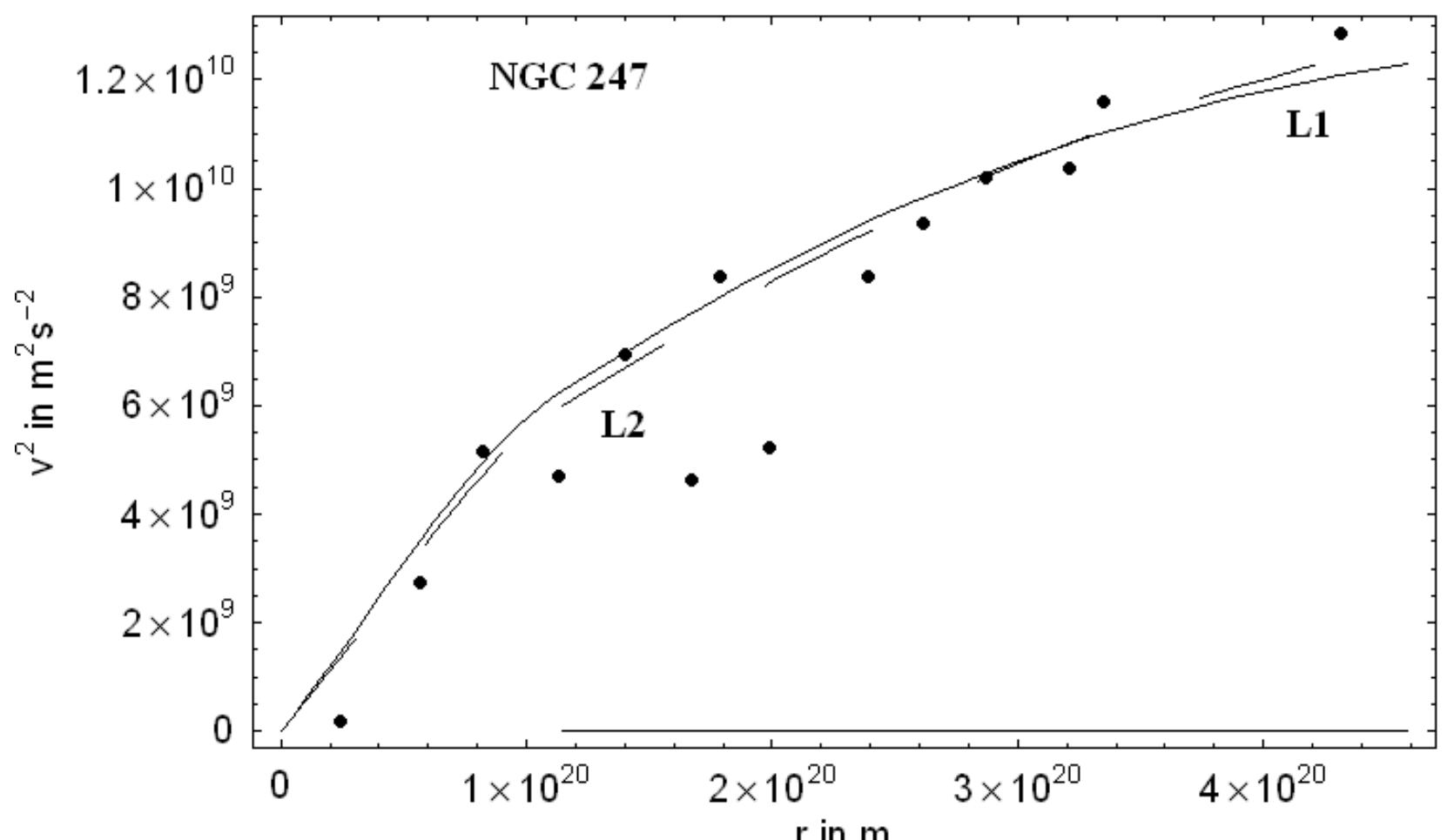

6: Rotation curves with a length scale as in Figure 1 and experimental points for the galaxy NGC 247 with $R_{0}=3.7 \mathrm{kpc}$ [24]. There is $M=0.09 \cdot 10^{10} M_{\text {sun }}$ and $M_{S} / M=33$ and 25 , respectively, whereas the polytropic amplitude varies slowly according to $\alpha \cdot 10^{-30} \approx 2.80$ and $2.96 \frac{\mathrm{s}^{2} \mathrm{~m}}{\mathrm{~kg}} / \mathrm{c}^{4}$, respectively, with a greater value as in other figures because of $R_{0}>R_{1}$. 


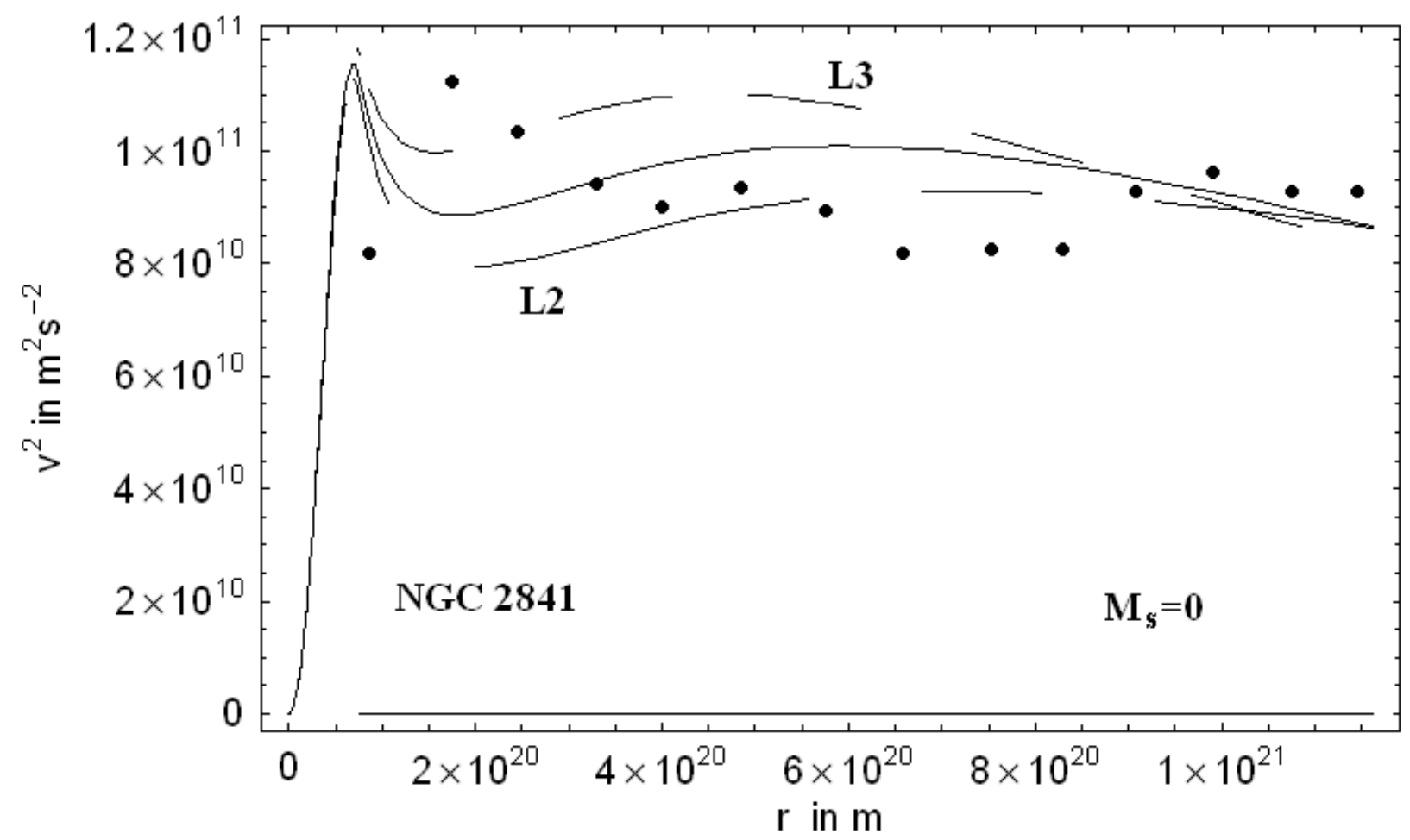

7: Rotation curves with a length scale $l=6 R_{1}$ (L2) and $l=5 R_{1}$ (L1) and $l=4 R_{1}$ (L3) with experimental points for the galaxy NGC 2841 with $R_{0}=2.45 \mathrm{kpc}$ [24]. The galaxy mass is given as $M=5.5 \cdot 10^{10} M_{\text {sun }}$ and the central mass is chosen as vanishing, whereas the polytropic amplitude varies slowly according to $\alpha \cdot 10^{-30} \approx 0.27,0.27$ and $0.28 \frac{\mathrm{s}^{2} \mathrm{~m}}{\mathrm{~kg}} / \mathrm{c}^{4}$, respectively. $R_{1}$ is the radius of the Milky Way.

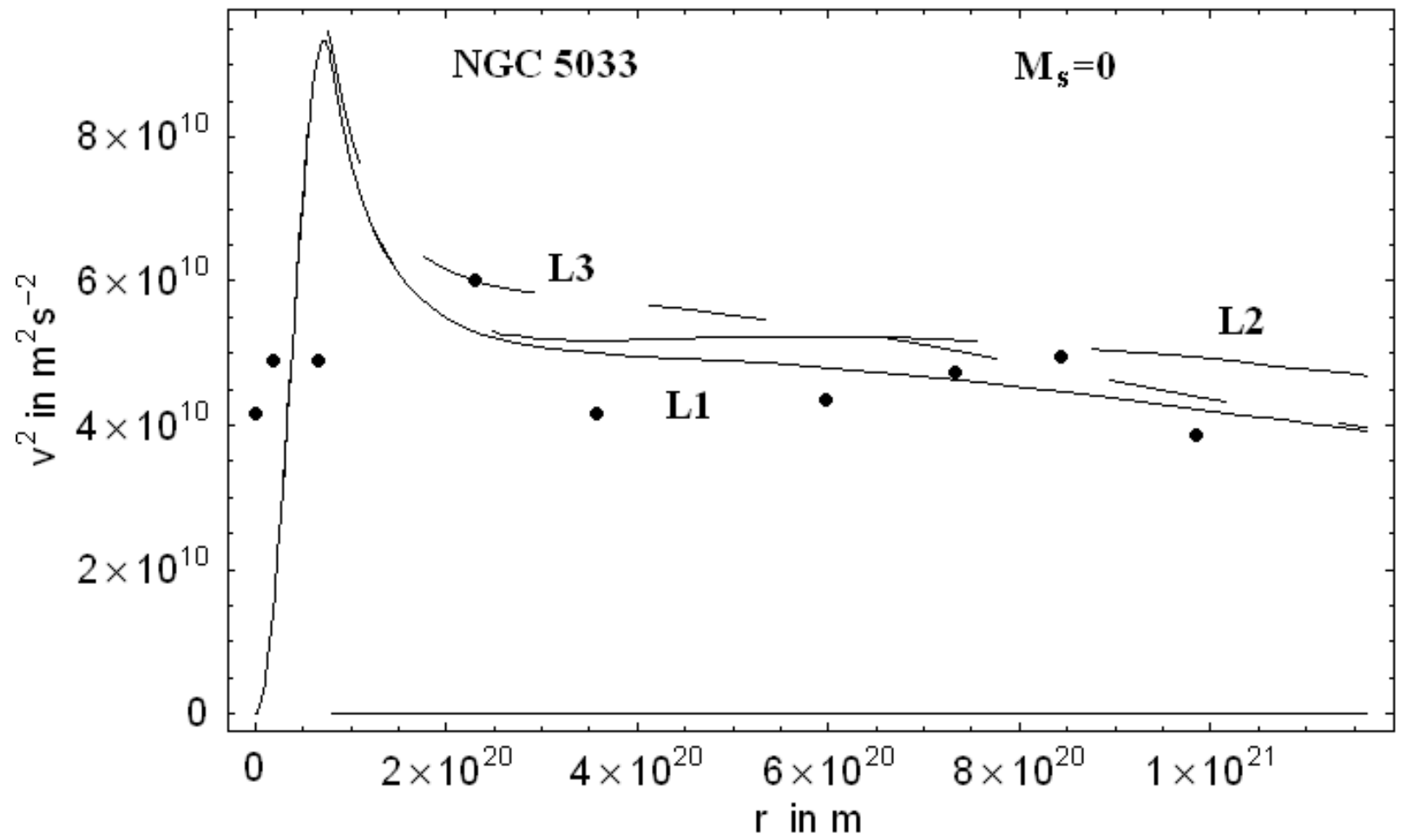

8: Rotation curves with a length scale as in Figure 7 and experimental points for the galaxy NGC 5033 [25] and radius $R_{0}=2.6 \mathrm{kpc}$. There is $M=5 \cdot 10^{10} M_{\text {sun }}$ with a vanishing central mass, whereas the polytropic amplitude varies slowly according to $\alpha \cdot 10^{-30} \approx 0.29,0.29$ and $0.30 \frac{\mathrm{s}^{2} \mathrm{~m}}{\mathrm{~kg}} / \mathrm{c}^{4}$, respectively. 


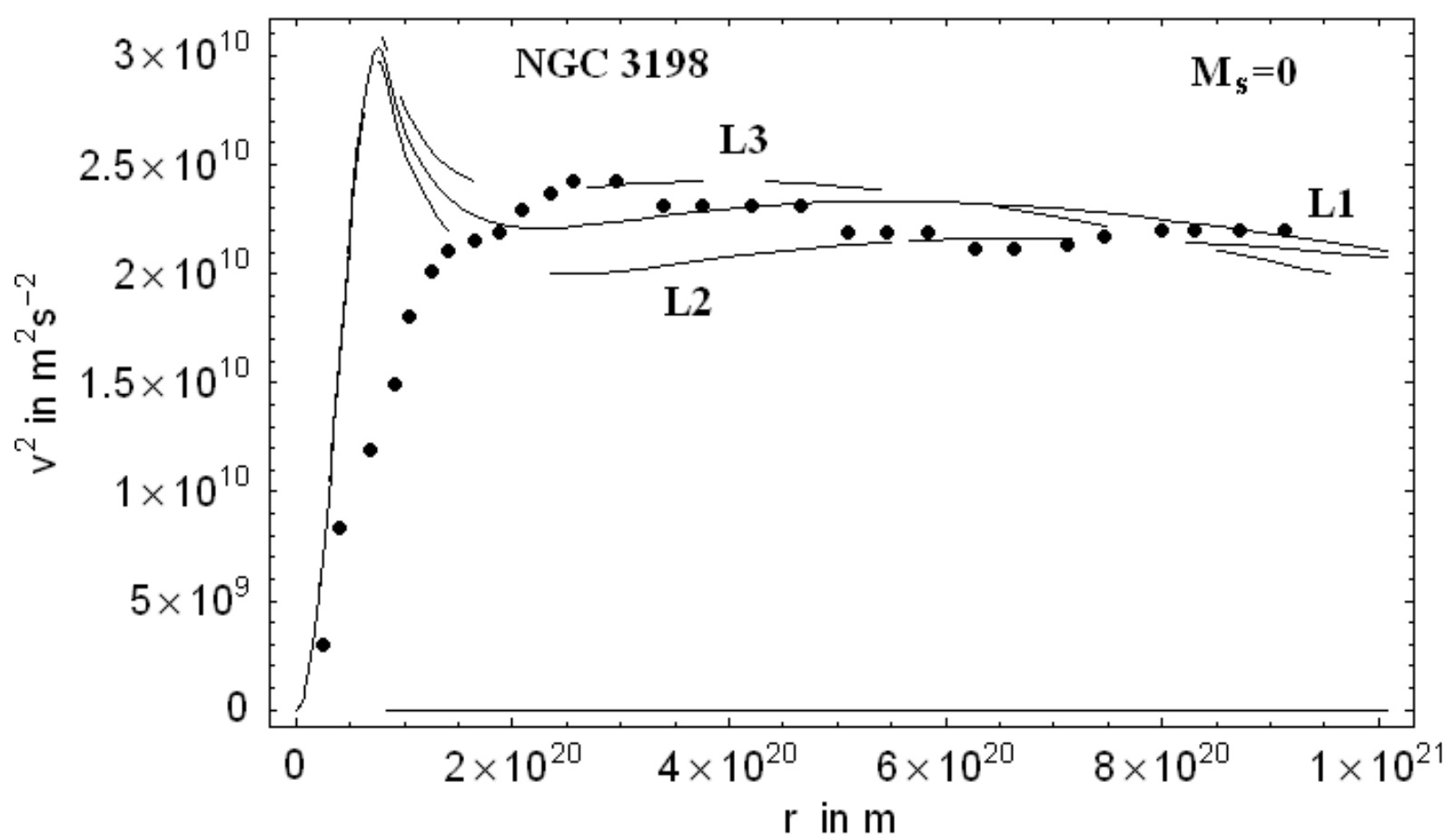

9: Rotation curves with a length scale as in Figure 7 and experimental points for the galaxy NGC 3198 with $R_{0}=2.68 \mathrm{kpc}$ [24]. There is $M=3 \cdot 10^{10} M_{\text {sun }}$ and $M_{S}=0$, whereas $\alpha \cdot 10^{-30} \approx 0.32 \frac{\mathrm{s}^{2} m}{\mathrm{~kg}} / \mathrm{c}^{4}$.

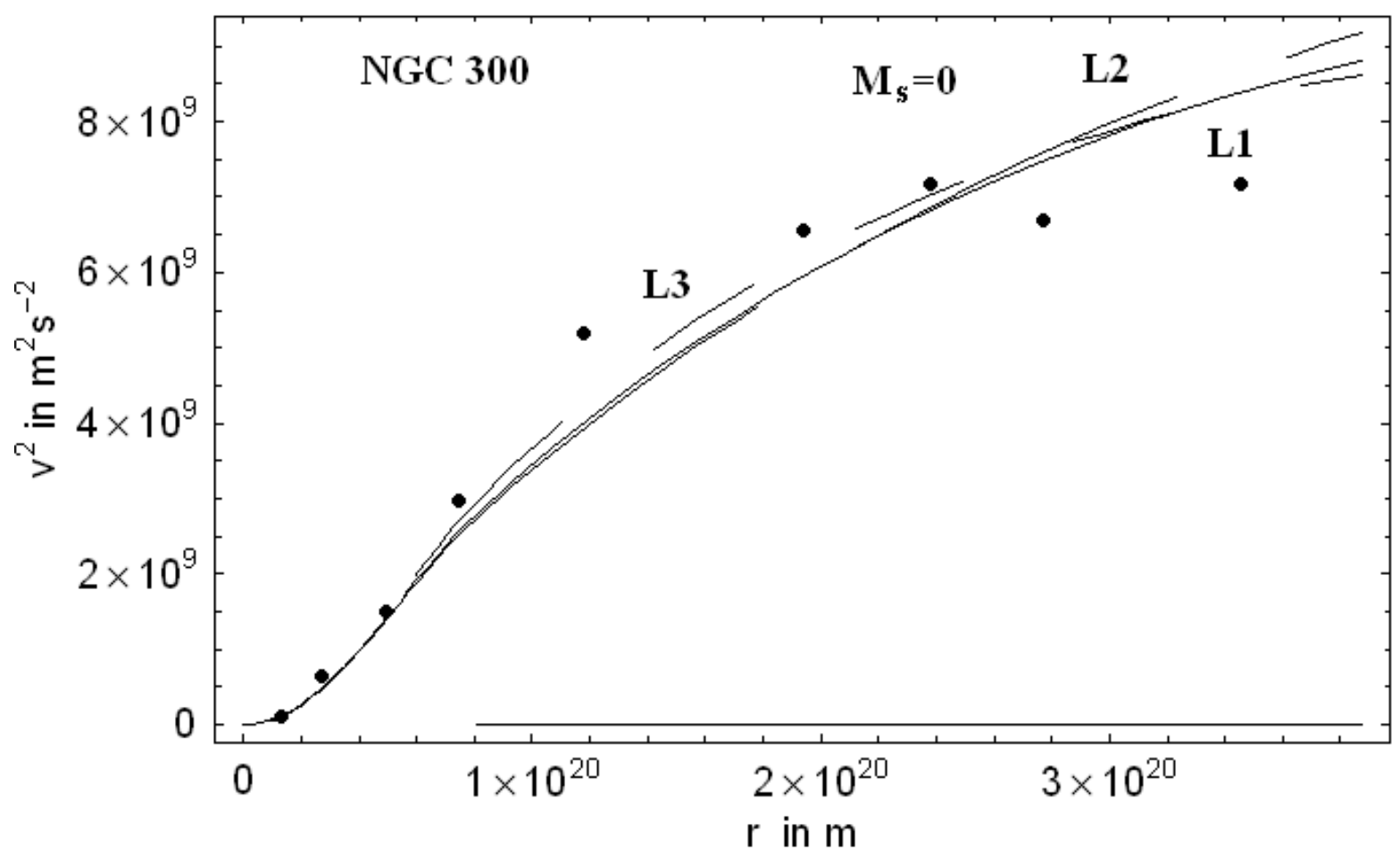

10: Rotation curves with a length scale as in Figure 7 and experimental points for the galaxy NGC 300 with $R_{0}=2.6 \mathrm{kpc}$ [24]. There is $M=0.055 \cdot 10^{10} M_{\text {sun }}$ and $M_{S}=0$, whereas the polytropic amplitude varies slowly according to $\alpha \cdot 10^{-30} \approx 0.63$, 0.64 and $0.67 \frac{\mathrm{s}^{2} m}{\mathrm{~kg}} / \mathrm{c}^{4}$, respectively. 


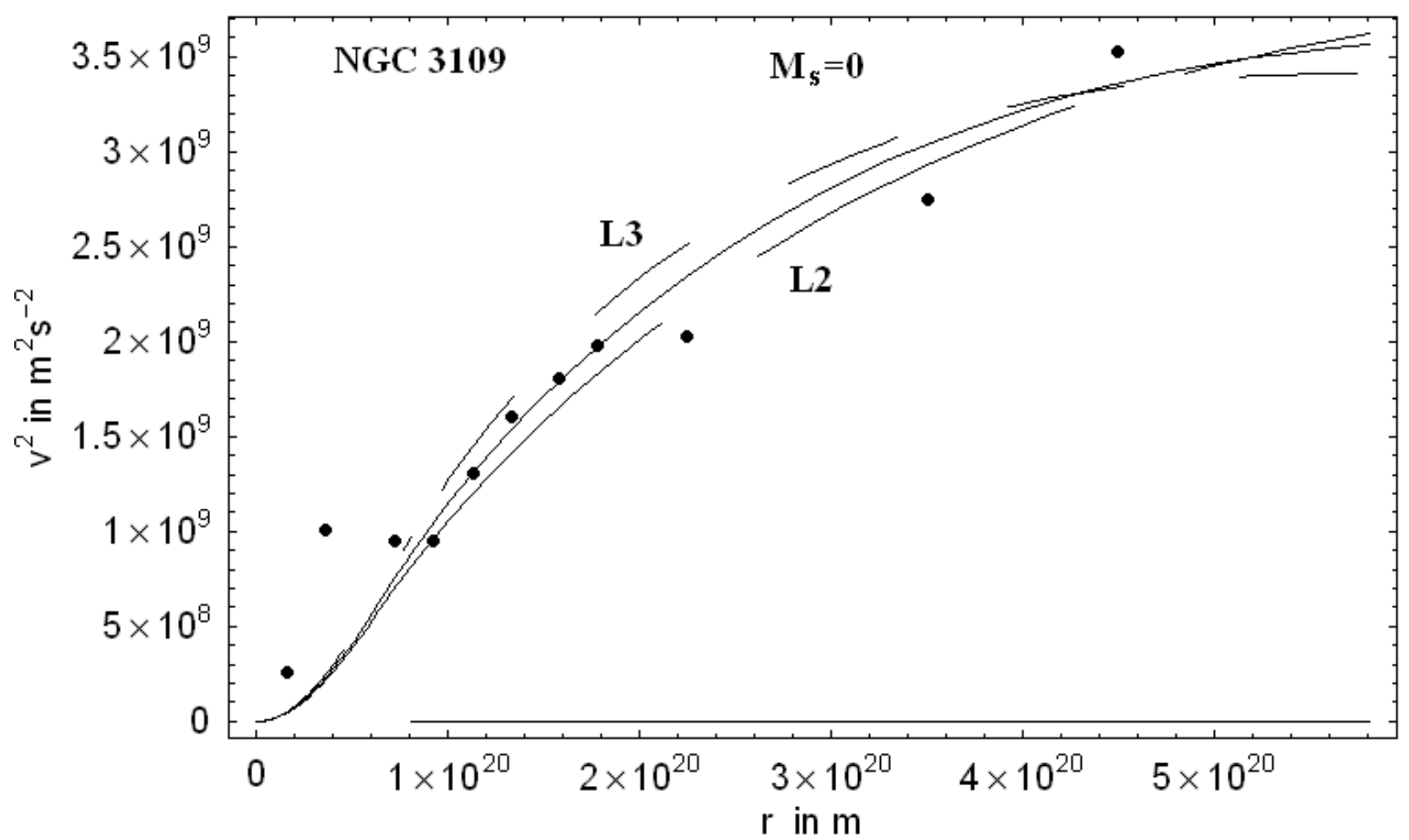

11: Rotation curves with a length scale as in Figure 7 and experimental points for the galaxy NGC 3109 with $R_{0}=2.5 \mathrm{kpc}$ [24]. There is $M=0.01 \cdot 10^{10} M_{\text {sun }}$ and $M_{S}=0$, whereas the polytropic amplitude varies slowly according to $\alpha \cdot 10^{-30} \approx$ $0.83,0.89$ and $0.97 \frac{\mathrm{s}^{2} \mathrm{~m}}{\mathrm{~kg}} / \mathrm{c}^{4}$, respectively.

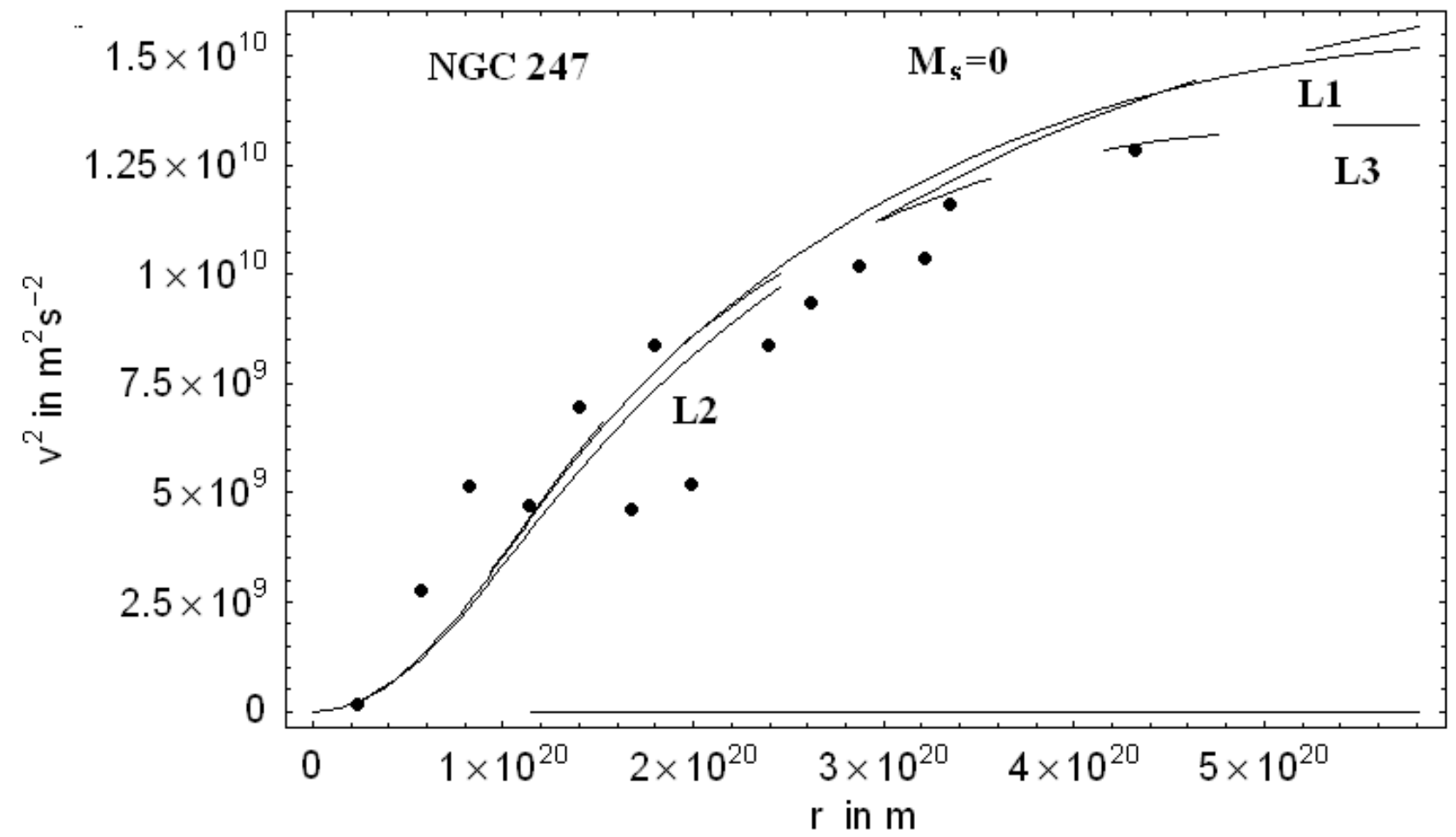

12: Rotation curves with a length scale as in Figure 7 and experimental points for the galaxy NGC 247 with $R_{0}=3.7 \mathrm{kpc}[24]$. There is $M=0.05 \cdot 10^{10} M_{\text {sun }}$ and $M_{S}=0$, whereas the polytropic amplitude varies slowly according to $\alpha \cdot 10^{-30} \approx 2.80$ and $2.96 \frac{\mathrm{s}^{2} \mathrm{~m}}{\mathrm{~kg}} / \mathrm{c}^{4}$, respectively (analog to Figure 6). 


\section{Conclusions}

The comparison of the theoretical rotation curves with the rotation curves for several galaxies indicates that the scalar-tensor theory with the Higgs Mechanism is able to explain and contribute to the explanation of their flatness, although in the case of high tangential velocities (see Fig. 1-3 and 7-9) the theoretical curves show a peak at $r=R_{0}$, which is observationally not always verified. Furthermore, it follows that a polytropic density distribution for galaxies is useful to achieve a satisfactory agreement between theoretical and empirical data, postulating or not postulating a central massive core for the galaxies. Nevertheless, the dynamics seem better explained by a non-vanishing central mass, which leads to the necessity of a higher galaxy mass. The central mass lies in the order of several galaxy masses, but is in conformity with this theory, since the mass interacts with the outer region only very weakly. This is in contrast to Newtonian mechanics, because in this scalar-tensor theory the effective gravitational coupling parameter is drastically diminished in the centre.

\section{References}

[1] van Albada, T.S.: ES, SP-183 (1988).

[2] Beckenstein, J. and Milgrom, M.: Astrophys. J. 186, 7 (1984).

[3] Bezares-Roder, N.M., Nandan, H. and Dehnen, H.: Int. J. Theor. Phys. 39(8) 1259 (2007).

[4] Brans, C. and Dicke, R.H.: Phys. Rev. 124, 925 (1961).

[5] Cervantes-Cota, J.L. and Dehnen, H.: Phys. Rev. D 51, 395 (1995).

[6] Cervantes-Cota, J.L. and Dehnen, H.: Nucl. Phys. B 442, 391 (1995).

[7] Cotsakis, S.: gr-qc/9712046 v1. Talk presented at the 8th Marcel Grossmann Meeting, Jerousalem June 22-27 (1997).

[8] Dehnen, H., Ghaboussi, F. and Schröder, J.: Wiss. Zeitschr. d. Friedrich-Schiller-Univ. Jena 39, 41 (1990).

Dehnen, H. and Frommert, H.: Int. J. Theor. Phys. 29(4), 361 (1990).

Dehnen, H., Frommert, H. and Ghabousssi, F.: Int. J. Theor. Phys. 29(6), 537 (1990).

[9] Dehnen, H. and Frommert, H.: Int. J. Theor. Phys. 30(7), 985 (1991).

[10] Dehnen, H., Frommert, H. and Ghaboussi, F.: Int. J. Theor. Phys. 31(1), 109 (1992).

[11] Dehnen, H. and Frommert, H.: Int. J. Theor. Phys. 32(7), 1135 (1993).

[12] Dehnen, H. and Obregón, O.: Astrophys. Sp. Sc. 14, 454 (1972).

[13] Einstein, A.: Phys. Zs. 14, 1260 (1913).

[14] Fäßler, A. and Jönsson, C.: Die Top Ten der schönsten physikalischen Experimenten; Rororo Science, Hamburg (2005).

[15] Fauser, B.: Gen. Relativ. Gravit. 33, 875 (2001).

[16] Gessner, E.: Astrophys. Sp. Sc. 194, 197 (1992).

[17] Hönl, H. and Dehnen, H.: Zs. f. Astrophys. 68, 181 (1968).

[18] Jordan, P.: Nature 164, 112 (1949).

Jordan, P.: Schwerkraft und Weltall; Friedr. Vieweg and Sohn Verlag, 2. Aufl., Braunschweig (1955).

[19] Jordan, P.: Zs. f. Astrophys. 68, 201 (1968).

[20] Matos, T., Guzmán, F.S. and Núńez, D.: Phys. Rev. D 62(061301R) (2000).

[21] Milgrom, M.: Astrophys. J. 270, 365 (1983).

[22] Ostriker, J.P. and Peebles, P.J.E.: Astrophys. J. 186, 467 (1973).

[23] Reasensberg, R.D. et al.: Astrophys. J. 234, L219 (1979).

Will, C.M.: Theory and Experiment in Gravitational Physics; University of Cambridge, Cambridge (1986).

[24] Sanders, R.H.: Astron. Astrophys. 154, 135 (1986).

[25] Sanders, R.H.: Astron. Astrophys. Rev. 2, 1 (1990).

[26] Zee, A.: Phys. Rev. Lett. 42(7), 417 (1979). 